\section{Per-operative malposition of foldable implants (IOL flip)}

Complications of cataract surgery relating to the insertion of intraocular lenses (IOLs) may occur per-operatively or in the early and late postoperative periods. The main per-operative complications associated with rigid, largediameter, posterior chamber lenses include posterior capsule rupture, zonular dehiscence and corneal endothelial trauma. Small-incision surgery usually involves inserting foldable or injectable lenses into the capsular bag through a continuous curvilinear anterior capsulotomy. Additional complications associated with these lenses include permanent deformation of loop haptics, damage to the optic ranging from surface lesions to full-thickness cracks in acrylic implants ${ }^{1}$ and IOL malposition. Malposition is usually thought of in terms of haptic location relative to the margin of the capsulorhexis or pupil. Asymmetric fixation of loop haptics is known, for example, to result in a higher incidence of post-operative lens decentration. ${ }^{2}$ This article describes in detail the occurrence, management and prevention of an illpublicised $^{3}$ malposition of foldable loop haptic lenses that we have termed an IOL flip.

\section{Case reports}

Case 1

A silicone loop IOL (AMO Phacoflex II SI-30NB) was folded parallel to the haptic axis, following successful phacoemulsification through a $3.2 \mathrm{~mm}$ temporal clear corneal incision in a left eye. It was then inserted through the corneal incision, with the trailing haptic pointing left (Fig. 1a). The left hand was pronated and the IOL released (Fig. 1b). The IOL became unstable and began inadvertently rotating anticlockwise because the folding forceps trapped the trailing haptic (Fig. 1c). Removing the forceps from the eye generated an anticlockwise torque sufficient to flip the IOL (Fig. 1d). The leading haptic was now in the bag, pointing the wrong way, while the trailing haptic lay external to the corneal incision (Fig. 1e).

\section{Case 2}

A silicone lens (AMO Phacoflex II SI-40NB) was folded by the assistant and handed to the surgeon. As the IOL was being inserted into the eye it was noted that it had been folded asymmetrically with the folding forceps too close to the edge of the IOL (Fig. 2a). Before the hand could be pronated in the anterior chamber, the IOL had flipped explosively (Fig. 2b).

\section{Case 3}

After uneventful surgery, an IOL introducing device, the Unfolder (AMO Phacoflex II, SofTip insertion system, Allergan), was used to inject an SI-40NB IOL into the capsular bag following viscoelastic injection into the anterior chamber. Though the leading haptic initially pointed correctly to the left (Fig. 3a), premature anticlockwise rotation of the handpiece, probably caused by poor visualisation due to an air bubble, resulted in a controlled IOL flip (Fig. 3b, c).

\section{Options}

When faced with this alarming intraoperative problem, what is the correct course of action? The IOL could be inserted into the capsular bag with the lens still malrotated. Although technically easy, this option is unsound for several reasons. The posterior angulation of the haptics would result in the IOL being displaced anteriorly in the visual axis. An unplanned myopic refraction may then create difficulty. Posterior capsule opacification occurs prematurely because of the lack of a barrier between the posterior capsule and the posterior surface of the IOL. ${ }^{2}$ While this is easily treated with a YAG laser, one must also consider in specific clinical situations the serious impact of retinal detachment, which is a recognised complication of capsulotomy. ${ }^{4}$ An inverted IOL also theoretically increases the risk of the anterior capsule prematurely fusing to the anterior surface of the IOL. Secondary glaucoma due to capsular blockage would then be a serious complication. ${ }^{5}$

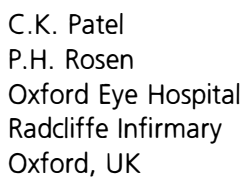

C.K. Patel, BSC, FRCOphth Oxford Eye Hospital Radcliffe Infirmary Woodstock Road Oxford OX2 6AW, UK

Fax: $+44(0) 1865794508$ e-mail: ckpatel@compuserve.com

This paper is derived from a video presentation at the Royal College of Ophthalmologists' annual congress in Glasgow, 1998

Received: 24 June 1998 Accepted: 18 December 1998 


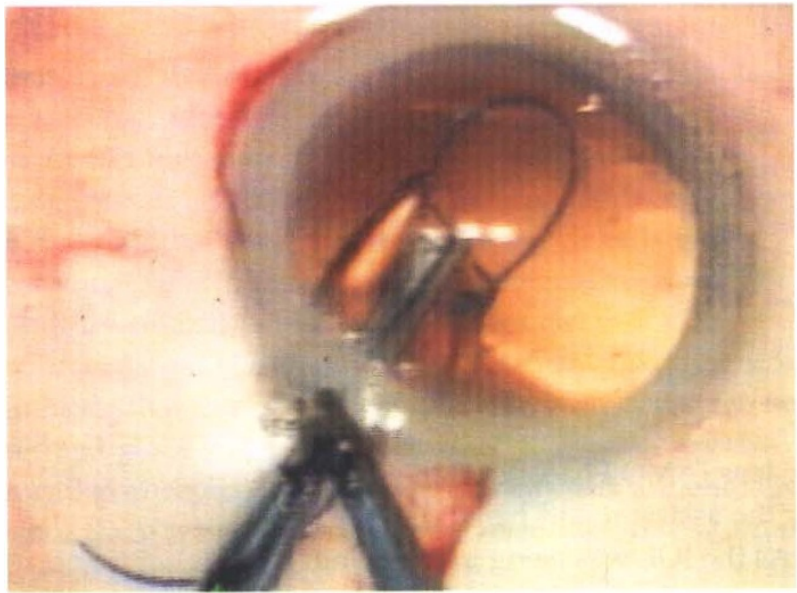

(a)

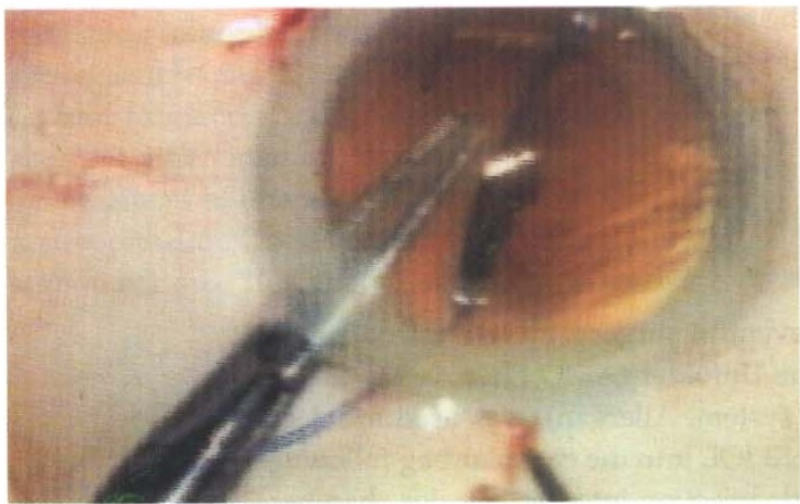

(c)

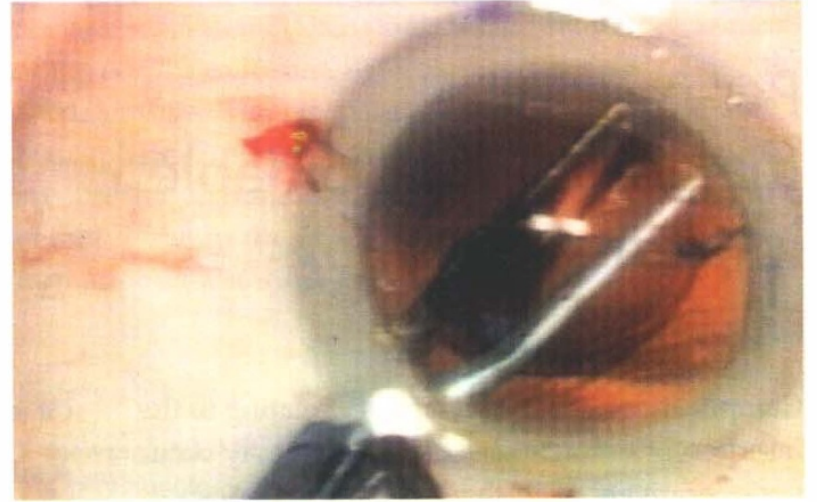

(b)

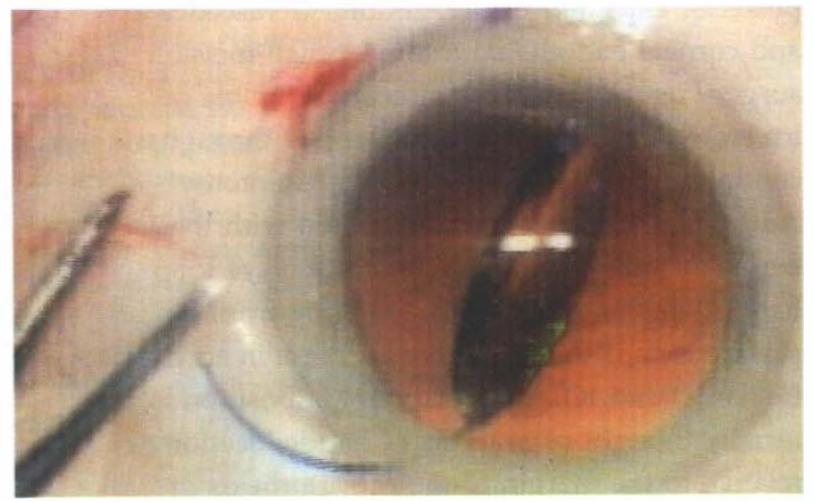

(d)

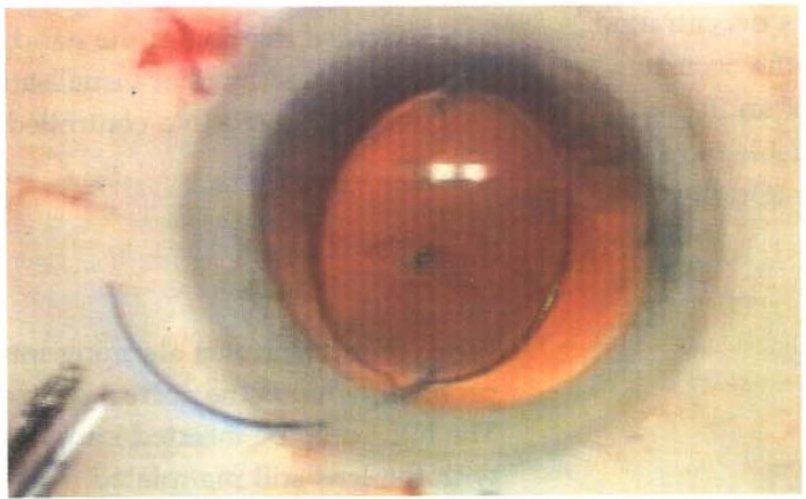

(e)

Fig. 1. Case 1. IOL flip occurring when using folding forceps.

The second surgical option is to exchange the IOL. The disadvantage of this approach is that the wound may need to be enlarged or the IOL may need to be divided in situ before being removed from the eye. It is possible to re-fold the IOL in the anterior chamber and remove it, though this usually requires an additional paracentesis. ${ }^{6}$

The third option, which in our opinion has the optimal risk-benefit ratio, is to flip the unfolded IOL

back in situ. The anterior chamber is first deepened with viscoelastic. An adhesive viscoelastic such as Viscoat is preferred for additional protection to the corneal endothelium. A clockwise torque is generated bimanually, either by two intraocular instruments or by one instrument inserted through the sideport and a forceps to manipulate the trailing haptic (Fig. 4a, b). In this way the optic rotates safely without injuring the endothelium. The trailing haptic is then inserted in the normal way. To date the procedure has not been associated with posterior capsule rupture and is associated with the expected good visual outcome (Fig. 4c).

IOL flips can be prevented in the following ways:

1. The IOL could be folded at $90^{\circ}$ to the haptic axis and the loops tucked into the folded optic. It can then be inserted into the bag in a one-step manoeuvre with a lower risk of a flip occurring. This can only be performed with flexible polypropylene loops that will not fracture when deformed. 


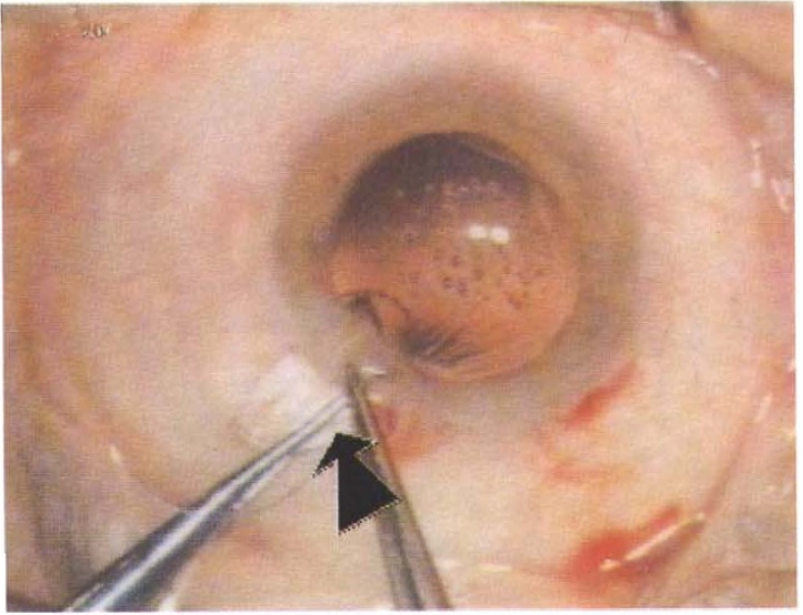

(a)

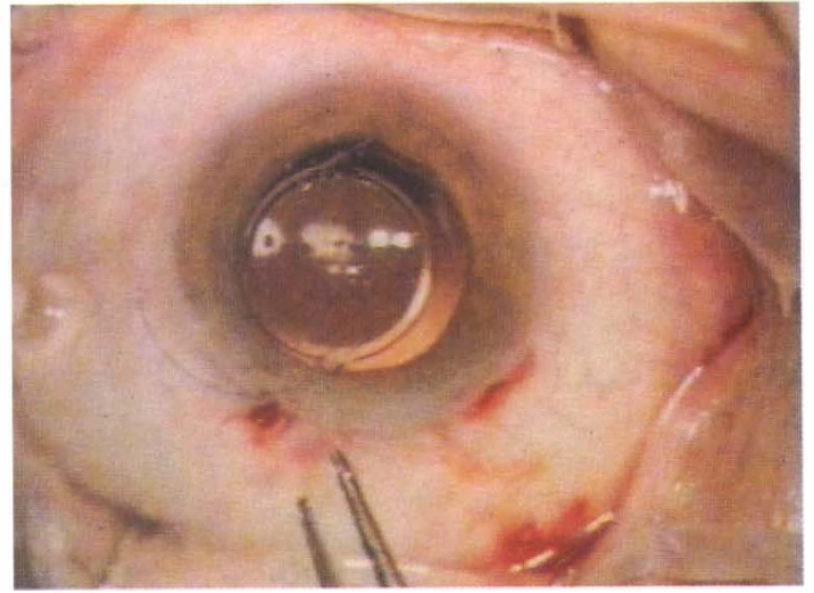

(b)

Fig. 2. Case 2. IOL flip occurring when using folding forceps.

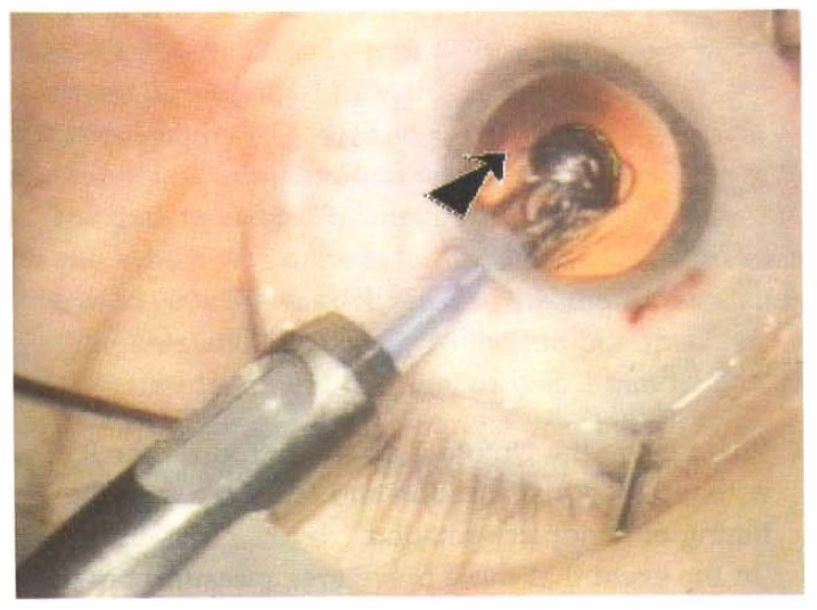

(a)

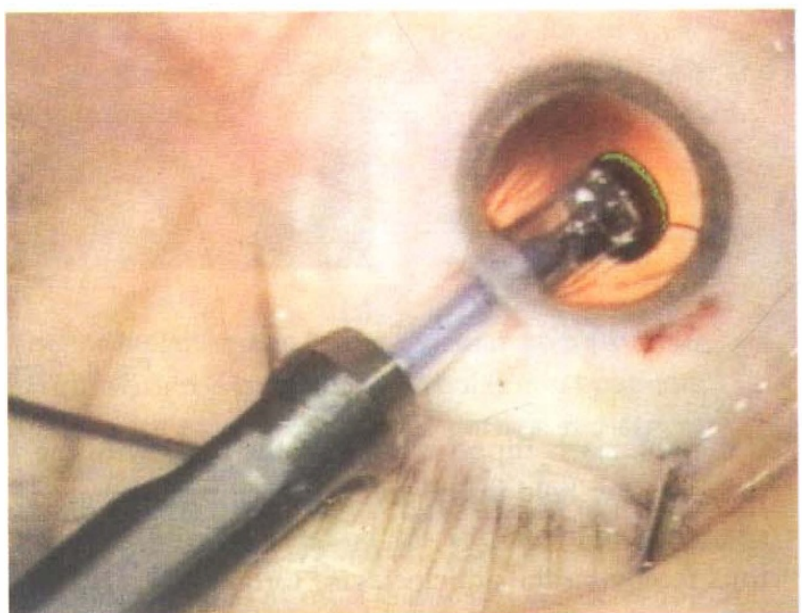

(b)

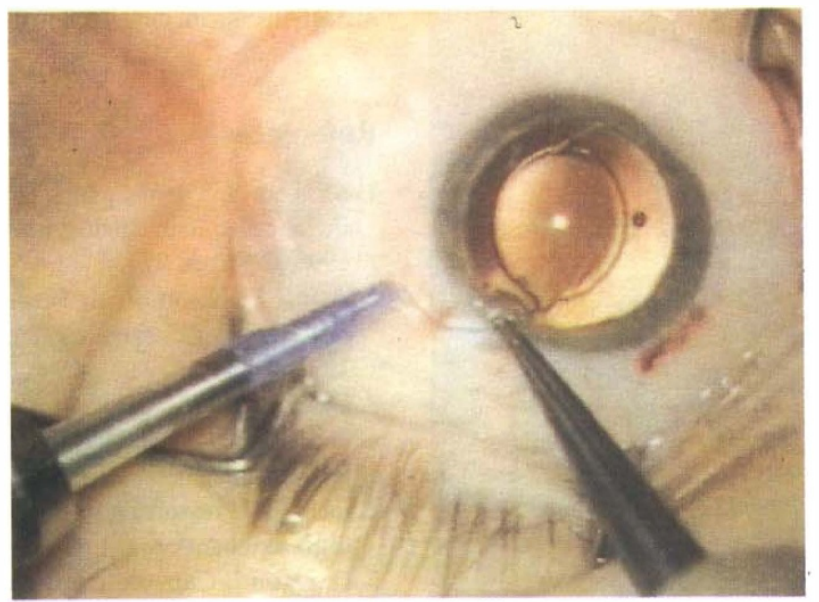

(c)

Fig. 3. Case 3. IOL flip occurring when using a lens introducer. 


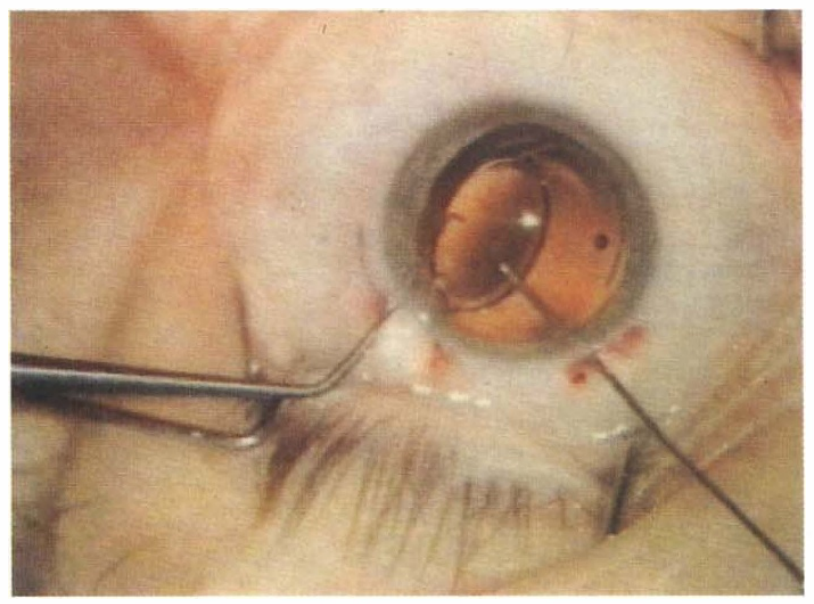

(a)

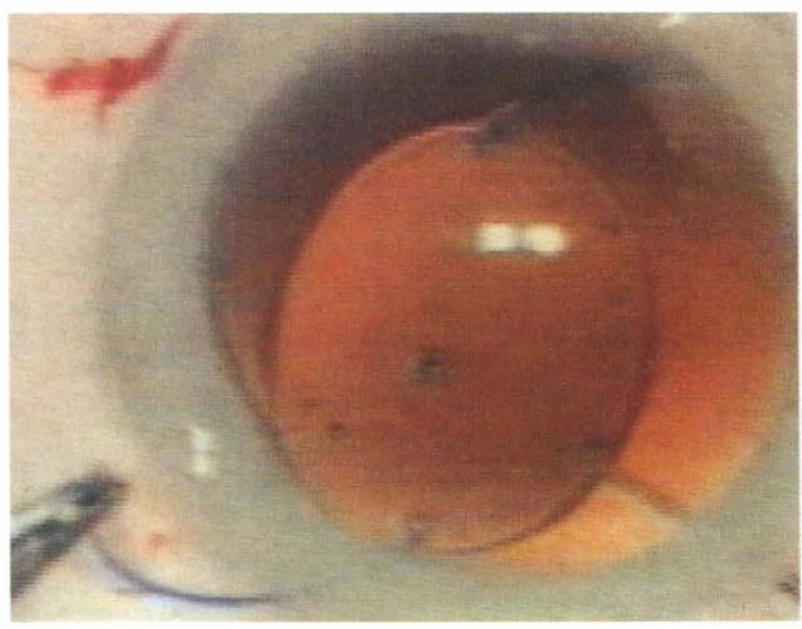

(b)

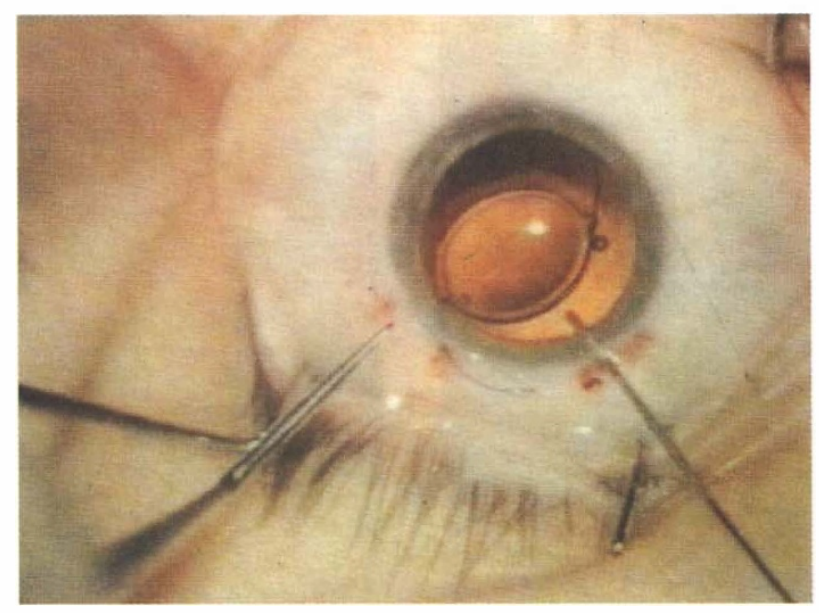

(c)

Fig. 4. Correcting an IOL flip.

2. If the IOL starts flipping, support the optic with the closed folder held in the anterior chamber so that the snagged trailing haptic can be released with forceps thus removing the anticlockwise torque (Fig. 5). The IOL can then be nudged and rotated to the correct orientation by the folding forceps.

3. Ensure correct folding of the IOL, if necessary by performing the procedure under the microscope.

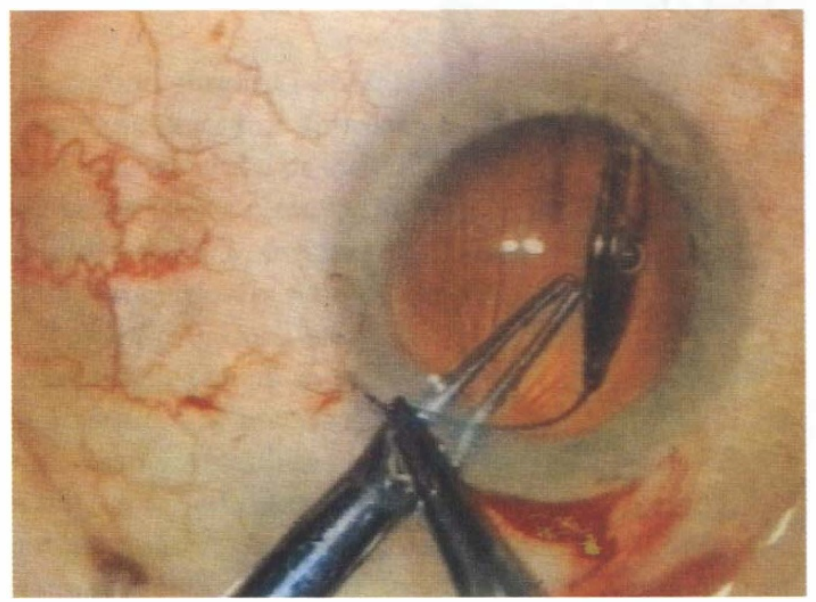

Fig. 5. Preventing an IOL flip.
4. When using the Unfolder do not introduce the IOL without good visualisation and take care with the timing of handpiece rotation.

In the event that these preventive measures are unsuccessful one should remain calm in the knowledge that the malpositioned IOL can safely be flipped back without compromising in any way the end result of small-incision cataract surgery.

\section{References}

1. Milazzo S, Turut $\mathrm{P}$, Blin $\mathrm{H}$. Alterations to the AcrySof intraocular lens during folding. J Cataract Refract Surg 1996;22(Suppl 2):1351-4.

2. Apple D, Solomon K, Tetz M, Assia E, Holland E, Legler U, et al. Posterior capsule opacification. Surv Ophthalmol 1992;37:73-116.

3. Olson R, Cameron R, Hovis T, Hunkeler J, Lindstrom R, Steinert R. Clinical evaluation of the Unfolder. J Cataract Refract Surg 1997;23:1384-9.

4. Jacobi F, Hessemer V. Pseudophakic retinal detachment in high axial myopia. J Cataract Refract Surg 1997;23:1095-102.

5. Davison J. Capsule contraction syndrome. J Cataract Refract Surg 1993;19:582-9.

6. Neuhann T. Intraocular folding of an acrylic lens for explantation through a small incision cataract wound. J Cataract Refract Surg 1996;22(Suppl 2):1383-6. 